\title{
A Note on Ocean Currents Produced by Sources and Sinks*
}

\author{
Takashi ICHIYE**
}

\begin{abstract}
Ocean currents produced by sources and sinks on a $\beta$-plane are treated. In presence of an eastern coast an isolated sink (or source) yields zonal flows only to the west of the sink (or source). The total zonal transport due to the sink (or source) is eastwards (or westwards). The pressure and velocity distributions induced by a point source are determined for the flow with frictional forces.
\end{abstract}

\section{Introduction}

GoLDSBROUGH (1933) discussed ocean currents produced by evaporation and precipitation, which are equivalent to distributed sources and sinks. Recently Stommel and Others (1958) studied a stationary planetary flow induced by combinationt of point and distributed sources and sinks, theoretically and experimentally. In this paper is discussed a mathematical model of currents on a $\beta$-plane produced by an isolated source or sink.

The model considered here gives a simple example illustrating characteristic features of the $\beta$-plane. The result may be applied to a stationary circulation in the deep layer where intense vertical currents serve as a source or sink, by supplying or substracting water in a small area.

\section{Mathematical Model}

Consider the ocean on a $\beta$-plane. The equations of motion become

$$
-f v=-\partial p / \partial x+F_{x}, f u=-\partial p / \partial y+F_{y}(1)
$$

in which $f$ is the Coriolis' parameter, $F_{\boldsymbol{x}}$ and $F_{y}$ are components of friction forces and $x$ and $y$ are coordinates pointing to the east and north, respectively. In presence of sources or sinks, the equation of continuity becomes

$$
\partial u / \partial x+\partial v / \partial y=-q \text {. }
$$

The vorticity equation derived from (1) is

$$
\beta v-f q=\partial F_{y} / \partial x-\partial F_{x} / \partial y \text {. }
$$

For a flow without friction forces this equation becomes

\footnotetext{
Received Feb. 8, 1960.

* Oceanographic Institute Contribution No. 150.

** Oceanographic Institute, Florida State University, Tallahassee, Florida.
}

$$
v=f^{-1}(\partial p / \partial x)=f \beta^{-1} q .
$$

Assume an eastern coast at $x=x_{1}$ where $u=0$ or $\partial p / \partial y=0$. The integration of (4) under this boundary condition gives

$$
p=f^{2} \beta^{-1} \int_{x_{1}}^{x} q d x
$$

in which $x<x_{1}$. The second equation of (1) leads to

$$
u=2 \int_{x}^{x_{1}} q d x+f \beta^{-1} \int_{x}^{x_{1}}(\partial q / \partial y) d x .
$$

In order to understand the meaning of these relations a schematical model shown in figure 1 is considered. An isolated sink (positive $q$ ), symmetrical about the $x$-axis, is assumed to have a north-south dimension of about $1000 \mathrm{~km}$. The equation (4) indicates that $v$ vanishes outside the sink area. According to (6), the zonal current begins at the eastern edge of the sink and its velocity becomes zonally constant to the west of the sink.

The first term in the right hand side of (6) gives an eastward flow to the west of the sink as shown by the curve (A) of figure 1 . The second term-a solid curve of (B)-represents the westward flow in the northern half and eastward flow in the southern half. Owing to northward increase of $f$ the profile indicates a north-south asymmetry, contrary to the symmetrical distribution corresponding to the constant $f$ (a broken line of (B) in figure 1). For a sink or source of the dimension of figure 1 the second term of (6) is predominant over the first term.

The total zonal transport due to a sink or source is obtained by integration of (6) with $y$ from $-\infty$ to $\infty$. The integral of the 
second term of (6) is given by

$$
\int_{x}^{x_{1}}\left\{\left.f \beta^{-1} q\right|_{-\infty} ^{\infty}-\int_{-\infty}^{\infty} q d y\right\} d x=-\int_{x}^{x_{1}} \int_{-\infty}^{\infty} q d y d x
$$

since $q$ is zero at $y= \pm \infty$. Therefore, we have

$$
\int_{-\infty}^{\infty} u d y=\int_{-\infty}^{\infty} \int_{x}^{x_{1}} q d x d y
$$

This equation indicates that the total water supply (or substraction) becomes the zonal transport from the source (or to the sink).

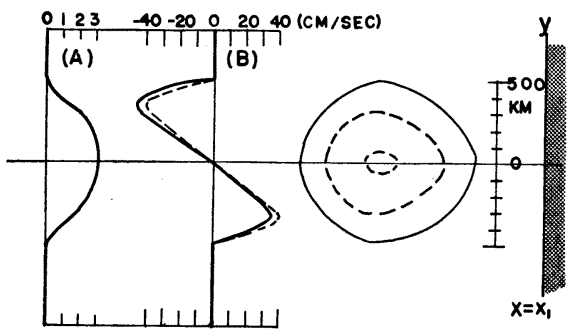

Fig. 1. Arrangements of an isolated sink and an eastern coast at $x=x_{1}$. The curves (A) and (B) represent meridional distributions of zonal currents given by the first and second term of the r.h.s. of the equation (6), respectively. (A broken curve of (B) is for $f=f_{0}$, the value of $f$ at $y=0$.)

Stommel and Others (1958) derived a conclusion that a point source (or sink) produces a simple westward (or eastward) flow to the west of the source (or sink), in contrast with a meridional structure of $u$ in this model for a source with a finite area. However, this model cannot be applied to a point source, because the second term of the right hand side of (6) becomes infinite as the dimension of the source diminishes to zero. Actually dissipative forces which are neglected in this model will be important as the area of the source decreases.

In presence of a western coast, the formal solution of (4) is the same as (5). However, the equations (1) are no longer valid near the western boundary owing to the importance of inertia terms neglected in (1) (MoRGAN, 1956; ICHIYe, 1959). In this paper, the effect of a western coast is not discussed.

\section{The flow with friction forces}

In order to obtain a circulation induced by a point source, friction forces are introduced.
Only for understanding the effect of friction, these forces may simply be taken as

$$
F_{x}=-k u, \quad F_{y}=-k v
$$

in which $k$ is assumed to be constant.

The equation of $p$ becomes

$$
k f \nabla^{2} p+\beta(f \partial p / \partial x-k \partial p / \partial y)=f\left(k^{2}+f^{2}\right) q .
$$

The value of $k$ was estimated by SтоммеL (1948) as $10^{-6}\left(\mathrm{sec}^{-1}\right)$ from comparison of observed and computed transports of the western current. This value indicates that $k \ll f$. Thus the equation (9) can be simplified as

$$
\nabla^{2} p+\beta k^{-1} \partial p / \partial x=f^{2} k^{-1} q .
$$

The solution of $p$ for a point source can be obtained by the Fourier integral method, in which $q$ is taken as

$$
\begin{gathered}
q=q_{0} \text { for }|x| \leq c \text { and }|y| \leq c^{\prime} ; \\
q=0 \text { elsewhere, }
\end{gathered}
$$

and $c$ is put to zero. The Fourier transform of $p$ about $y$ becomes

$$
p=(2 \pi)^{-1} \int_{-\infty}^{\infty} e^{i a \eta} P(x, a) d a .
$$

The equation for $P$ is given by

$$
\partial^{2} P / \partial x^{2}+2 h \partial P / \partial x-a^{2} P=f^{2} k^{-1} Q
$$

in which

$$
Q(x, a)=\int_{-\infty}^{\infty} e^{-i a y} q(x, y) d y
$$

and $2 b=\beta k^{-1}$.

Under the boundary condition at an eastern coast that

$$
P=0 \quad \text { at } x=x_{1},
$$

the general solution of (13) is expressed by

$$
\begin{aligned}
P= & A\left\{\exp \delta_{1}\left(x-x_{1}\right)-\exp \delta_{2}\left(x-x_{1}\right)\right\} \\
& +\frac{1}{2} f^{2}(\lambda k)^{-1} \int_{x_{1}}^{x} Q\left\{e^{\delta_{1}(x-t)}-e^{\delta_{2}\left(x-t^{t}\right.}\right\} d t
\end{aligned}
$$

in which

$$
\delta_{1}=-b+\lambda, \delta_{2}=-b-\lambda, \lambda^{2}=a^{2}+b^{2} .
$$

The other boundary condition at a western coast may determine the constant $A$ of (16). In this model, instead, the condition that

$$
P=\text { finite } \quad \text { at } x=-\infty
$$

is considered. The function $Q$ corresponding to the distribution of $q$ given by (11) is

$$
\begin{array}{ll}
Q=Q_{0}=2 q_{0} a^{-1} \sin a c^{\prime}, & \text { for }|x| \leq c ; \\
Q=0, & \text { for }|x| \geq c
\end{array}
$$


The boundary condition (18) and the relation (19) determines $A$ as

$$
\begin{aligned}
A & =f^{2} Q_{0}\left(\delta_{2} \lambda k\right)^{-1} \sinh \delta_{2} c \cdot \exp \delta_{2} x_{1} \\
& =A_{0} \exp \delta_{2} x_{1} .
\end{aligned}
$$

Thus the solution (15) with $A$ given by (20) is written as

$$
\begin{aligned}
& P=A_{0}\left\{\exp \left(\delta_{1} x-2 \lambda x_{1}\right)-\exp \delta_{2} x\right\}, \text { for } x \geq c ; \\
& P=\left\{A_{0} \exp \left(-2 \lambda x_{1}\right)-B_{0}\right\} \exp \delta_{1} x,
\end{aligned}
$$$$
\text { for } x \leq-c
$$

in which

$$
B_{0}=f^{2} Q_{0}\left(\delta_{1} \lambda k\right)^{-1} \sinh \delta_{1} c .
$$

For numerical values of $k=10^{-6}\left(\mathrm{sec}^{-1}\right)$ and $\beta=2 \times 10^{-13}\left(\mathrm{~cm}^{-1} \mathrm{sec}^{-1}\right), b$ is equal to $10^{-7}$ $\left(\mathrm{cm}^{-1}\right)$. Thus when the source is apart from the eastern coast by more than $100 \mathrm{~km}$, we can put $\exp \left(-2 \lambda x_{1}\right) \ll 1$. Then (21) can be written as

$$
\begin{aligned}
& P=-A_{0} \exp \delta_{2} x, \quad \text { for } x \geq c ; \\
& P=-B_{0} \exp \delta_{1} x, \quad \text { for } x \leq-c .
\end{aligned}
$$

Further, if $c$ is small enough to put $\delta_{2} c \ll 1$, (23) is expressed by

$$
\begin{array}{r}
P=-f^{2} Q_{0} c(\lambda k)^{-1} \exp (-b x-\lambda|x|), \\
\text { for }|x| \geq c
\end{array}
$$

(In a model with a western coast at $x=x_{1}$ $(<0)$, the formal solution of (13) is again given by (16). The condition that $P$ is finite at $x=\infty$ determines the value of $A$ as

$$
A=-B_{0} \exp \delta_{1} x_{1} \text {. }
$$

Corresponding to (21), we have

$$
\begin{aligned}
& P=\left(\mathrm{B}_{0} \exp 2 \lambda x_{1}-A_{0}\right) \exp \delta_{2} x, \quad \text { for } x>c ; \\
& P=B_{0}\left\{\exp \left(\delta_{2} x+2 \lambda x_{1}\right)-\exp \delta_{1} x\right\}, \text { for } x<-c
\end{aligned}
$$

Therefore, when $\lambda\left|x_{1}\right| \gg 1$, the same approximation of $P$ for $x>c$ as in (23) is valid. However, for $x<-c$ the approximation similar to (23) is valid except near the western coast, where the term $\exp \left(\delta_{2} x+2 \lambda x_{1}\right)$ becomes important. This corresponds to the effect of intensification of currents due to the western coast [ICHIYE, 1958].)

Under the condition that $c^{\prime}$ is small enough to insure $\sin a c^{\prime} \approx a c^{\prime}$, the Fourier integral (12) becomes

$$
p=-2 f^{2} q_{0} c c^{\prime}(\pi k)^{-1} \int_{0}^{\infty} \cos a y e^{\delta_{1} x} \lambda^{-1} d a .
$$

This integral is expressed by a modified
Bessel function $K_{0}(x)$ as (ERDELYI et al, 1954)

$$
p=-2 f^{2} q_{0} c c^{\prime}(\pi k)^{-1} e^{-b x} K_{0}(b r)
$$

in which $r^{2}=x^{2}+y^{2}$. This equation represents the Green function for the equation (10) (Veronis, 1958). However, it should be noted that the dimensions of the source $c$ and $c^{\prime}$ must be less than $100 \mathrm{~km}$ for $k=10^{-6}\left(\mathrm{sec}^{-1}\right)$ (or less than $1000 \mathrm{~km}$ for $k=10^{-5}$ ) so that the approximation leading from (23) to (24) and (25) might be valid.

The geostrophic component of a zonal velocity is expressed by

$$
\begin{gathered}
u_{g}=-f^{-1}(\partial p / \partial y)=4 b S e^{-b x}\left\{2 K_{0}(b r)\right. \\
\left.-f y(2 k r)^{-1} K_{1}(b r)\right\}
\end{gathered}
$$

in which $S=q_{0} c c^{\prime} / \pi$. The profiles of $u_{g}$ at $x$ $=-500 \mathrm{~km}$ and $-1000 \mathrm{~km}$ are plotted with a unit of $4 b S$ in figures 2(A) and 2(B) for $k$ $=10^{-6}$ and $10^{-5}\left(\mathrm{sec}^{-1}\right)$, respectively. These profiles indicate that $u_{g}$ is westward in the northern half and eastward in the southern half for positive $q_{0}$ (sink), as is the case of
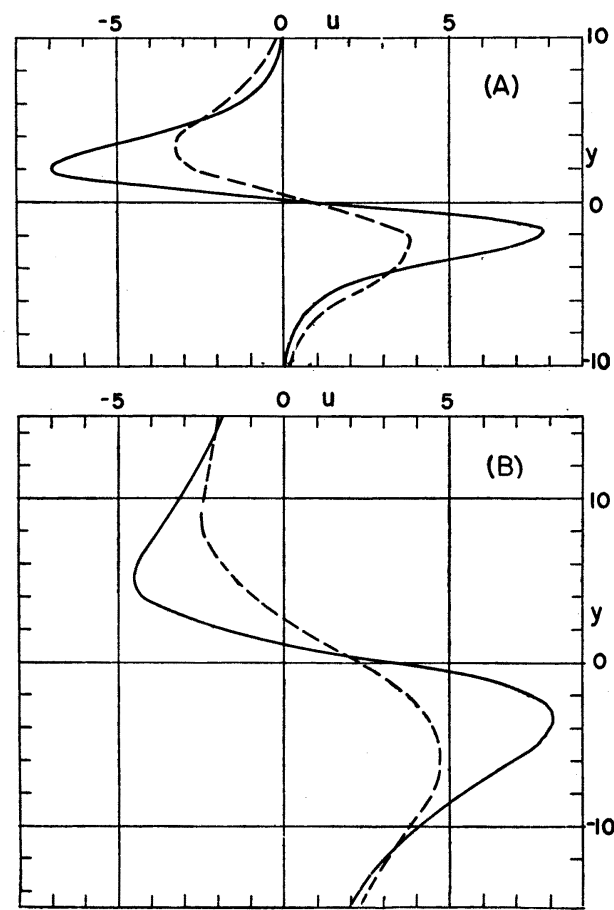

Fig. 2. Distributions of $u_{g}$ (in a unit of $4 b S$ ) against $y$ (in $100 \mathrm{~km}$ ) at $x=-500 \mathrm{~km}$ (full lines) and at $x=-1000 \mathrm{~km}$ (broken lines). Figure (A) is for $k=10^{-6}$ and (B) for $k=10^{-5}$ $\left(\sec ^{-1}\right)$. 
figure 1. The north-south gradient and maximal absolute value of $u_{g}$ decrease as the distance from the source increases and as the value of $k$ increases. As usually is the case, friction forces tend to decrease the gradient and absolute values of velocity. The pressure field given by (26) is plotted for $k=10^{-6}$ in figure $3(\mathrm{~A})$, which shows rapid decrease of $p$ to the east of the source.

The total zonal transport of a geostrophic current is given by

$$
\int_{-\infty}^{\infty} u_{g} d y=-\left.f^{-1} p\right|_{-\infty} ^{\infty}-\int_{-\infty}^{\infty} \beta f^{-2} p d y \text {. }
$$

Since $p$ vanishes at $y= \pm \infty,(28)$ becomes

$$
\int_{-\infty}^{\infty} u_{g} d y=8 b S e^{-b x} \int_{0}^{\infty} K_{0}(b r) d y .
$$

The formula of a modified Bessel function (ERDELyi et al, 1953) gives

$$
\int_{0}^{\infty} K_{0}\left[b\left(x^{2}+y^{2}\right)^{1 / 2}\right] d y=(\pi / 2 b) \exp (-b|x|) .
$$

Therefore, for $x<0$, we have

$$
\int_{-\infty}^{\infty} u_{g} d y=4 c c^{\prime} q_{0}
$$

This relation indicates that the total water supply from the source is carried away by a geostrophic component of zonal currents.

When the source or sink is due to the vertical current $w$ at the top of the deep layer of a thickness $h, q_{0}$ is equal to $w / h$. Using $c=c^{\prime}=10^{7}(\mathrm{~cm}), w=\pi \times 10^{-4}(\mathrm{~cm} / \mathrm{sec})$ and $h=2$ $\times 10^{5}(\mathrm{~cm})$, we have $4 b S=2 \times 10^{-8} k^{-1}$. This gives the maximal value of $u_{g}$ of $0.16(\mathrm{~cm} /$ $\mathrm{sec}$ ) at the distance of $500 \mathrm{~km}$ west of the sink, for $k=10^{-6}\left(\mathrm{sec}^{-1}\right)$. The total water supply $4 c c^{\prime} w$ from the source is equal to 0.126 $\times 10^{6}\left(\mathrm{~m}^{3} / \mathrm{sec}\right)$.

The water supply at the source in the North Atlantic postulated by STommel (1958) in his scheme of deep circulation $20 \times 10^{6}\left(\mathrm{~m}^{3}\right.$ $/ \mathrm{sec})$ leads to the vertical velocity of $5 \times 10^{-2}$ $(\mathrm{cm} / \mathrm{sec})$ for the same values of $c$ and $c^{\prime}$ as above. Then the maximal $u_{g}$ at $500 \mathrm{~km}$ west of the source is about $25(\mathrm{~cm} / \mathrm{sec})$ for the same magnitudes of $k$ and $h$.

\section{A point source at an eastern coast}

The pressure distribution for a model with a point source at an eastern coast can be obtained by putting $x_{1}=c$ in the second equation of (21) and letting $c \rightarrow 0$. When the third and higher powers of $c$ in the expansions of $A_{0}$ and $B_{0}$ in (21) are neglected, we have

$$
P=-f^{2} k^{-1} c^{\prime} c^{2} q_{0} \exp \delta_{1} x \text {, for } x<-c
$$

The Fourier integral for $p$ becomes

$$
p=-4 f^{2}(k \pi)^{-1} c^{\prime} c^{2} q_{0} e^{-b x} \int_{0}^{\infty} \cos a y e^{-\lambda|x|} d a .
$$

This is again expressed by a modified Bessel function (ERDÉLYI et al, 1954) like

$$
p=-4 f^{2}(k \pi)^{-1} c^{\prime} c^{2} q_{0} b|x| r^{-1} e^{-b x} K_{1}(b r) \text {. }
$$

The distribution of $p$ computed by (34) is plotted in figure $3(\mathrm{~B})$ with a unit of $0.4 f_{0}{ }^{2}(k \pi)^{-1}$ $\cdot c^{\prime} b c^{2} q_{0}$, in which $f_{0}$ is the value of $f$ at $y$ $=0$. If $b c=\frac{1}{2}$ (or $c=50 \mathrm{~km}$ for $k=10^{-6}$ ), this unit is equal to the one used in figure $3(\mathrm{~A})$. The pressure distribution of (34) is similar to figure $3(\mathrm{~A})$ except near the source, though the equations are different. The source in figure $3(\mathrm{~B})$ forms a singular point to which all isobars converge, but it is a singular point corresponding to $p=\infty$ in figure $3(\mathrm{~A})$.

The total zonal transport of a geostrophic component is given by

$\int_{-\infty}^{\infty} u_{g} d y=8(\pi)^{-1} b^{2} c^{\prime} c^{2} q_{0}|x| e^{-b x} \int_{-\infty}^{\infty} r^{-1} K_{1}(b r) d y$.

Again, from the relation of a modified Bessel function (ERDELYI et al., 1953), we have

$$
\int_{0}^{\infty}\left(t^{2}+z^{2}\right)^{-1 / 2} K_{1}\left[a\left(t^{2}+z^{2}\right)^{1 / 2}\right] d t=\pi(2 a z)^{-1} e^{-a|z|},
$$

(35) is written as

$$
\int_{-\infty}^{\infty} u_{g} d y=4 b c^{\prime} c^{2} q_{0}
$$

which is different from the total supply $4 c c^{\prime} q_{0}$ from the source. In order to obtain a zonal geostrophic transport equal to this supply, the higher powert of $c$ must be taken into account in the expansion of (21).

The water from the source is transported neither by a non-geostrophic zonal component nor by meridional currents through the sides $\mathrm{A}$ and $\mathrm{C}$ of figure 4 , as the $y$-coordinates $y_{0}$ and $-y_{0}$ of $\mathrm{A}$ and $\mathrm{C}$ approach to $\pm \infty$, respectively.

From (1) and (8), the velocity components 
are given by

$$
\begin{aligned}
u= & -f(\partial p / \partial y+k \partial p / \partial y)\left(f^{2}+k^{2}\right)^{-1} \\
& v=(-k \partial p / \partial y+f \partial p / \partial y)\left(f^{2}+k^{2}\right)^{-1} .
\end{aligned}
$$

First, it will be proved that

$$
\int_{A} v d x, \quad \int_{C} v d y \rightarrow 0 \text { as } y_{1} \rightarrow \infty .
$$

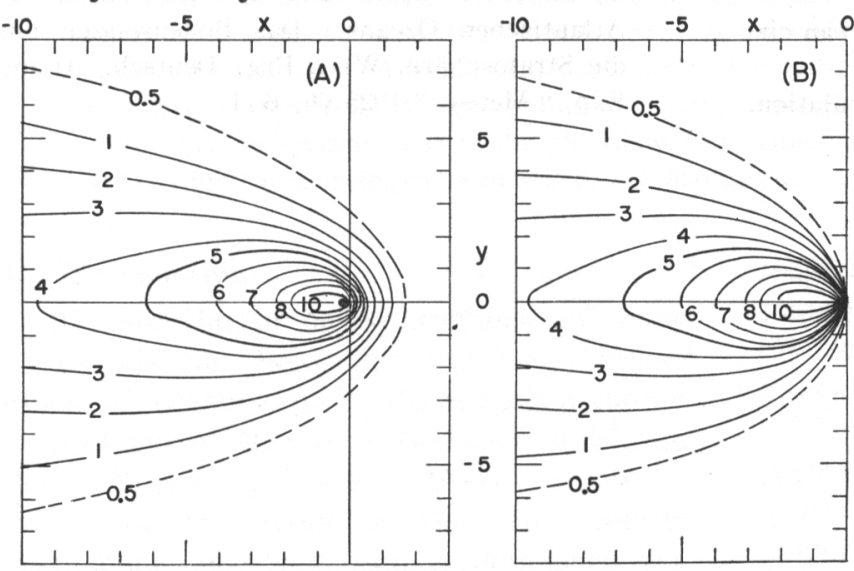

Fig. 3. Pressure patterns: (A) computed from (26) (in a unit of $\left.-0.2 f_{0}{ }^{2}(k \pi)^{-2} c c^{\prime} q_{0}\right)$ and (B) computed from (34) (in a unit of $\left.-0.4 f_{0}^{2}(k \pi)^{-1} b c^{2} c^{\prime} q_{0}\right)$. The distances in $x$-and $y$-direction are shown in $100 \mathrm{~km}$.

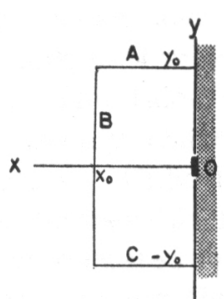

Fig. 4. The paths of integration around a point source for determining a net transport.
Substitution of (34) into the second equation of (38) gives

$$
v \sim \exp (-b|y|) F(x, y)
$$

in which $F(x, y)$ is an algebraic function of $y$, when $|y|$ is sufficiently large, as easily derived from the asymptotic formula of modified Bessel functions. Then, the relation (39) is proved to be valid for a finite value of $x_{0}$, the $x$-coordinate of the side $B$ (Fig. 4).

Since $f^{2} \gg k^{2}$, a non-geostrophic component of zonal currents of (38) is given by $u_{n}$ $=-k f^{-2}(\partial p / \partial x)$. The integration of $u_{n}$ along the side $\mathrm{B}$ gives

$$
\begin{aligned}
\int_{-\infty}^{\infty} u_{n} d y=4 \pi^{-1} c^{\prime} c^{2} b q_{0} \frac{\partial}{\partial x} \\
\times\left[|x| e^{-b x} \int_{-\infty}^{\infty} r^{-1} K_{1}(b r) d y\right]
\end{aligned}
$$

From the relation (36), the expression under partial differentiation about $x$ is equal to $\pi$. Thus the right hand side of (40) vanishes. It is proved that the non-geostrophic component of zonal currents does not transport a net amount of water. The same argument can be applied in the previous section to prove that only a geostrophic zontal component to the west of the source transports the net water supplied by the source.

\section{Conclusion}

The analysis for a point source with friction as well as for an isolated but finite-dimension source gives different distributions of a zonal velocity from the experiment in a rotating basin by Stommel and Others (1958). Such discrepancy is reasonable, because the condition at the slots which supply water into the basin of the experiment may be quite different from the theoretical model treated here.

Neither the circulation pattern induced by a source at the eastern coast has similarity to the spread of the Mediterranean water into the North Atlantic indicated by salinity distribution (Wüst, 1936). However, the vortices with a length scale of $1000 \mathrm{~km}$ found at the intermediate depth (Montgomery, 1938) may be attributed to a permanent sink or source in a small, limited area.

\section{Acknowledgement}

The author expresses his thanks to Dr. Sidney W. Fox and Dr. F. C. W. Olson for their encouragements throughout the present work.

\section{References}

ERDÉlyI et al, 1953: Higher transcendental functions II. New York, McGraw-Hill.

ERDÉLYI et al, 1954: Tables of integral transformations I. New York, McGraw-Hill.

Goldsbrough, G. R., 1933: Ocean currents produced by evaporation and precipitation. Proc. Roy. Soc. A, 512-517.

ICHIYE, T., 1958: The response of a stratified, bounded ocean to variable wind stresses. 
Oceanog. Mag. 10 (1), 19-63.

ICHIYE, T., 1959: On the response of western boundary currents to variable wind stresses. Journ. Geophys. Res. 64 (2), 175-189.

MONTGOMERY, R. B., 1938: Circulation in upper layers of Southern North Atlantic deduced with use of isentropic analysis. Papers in Phys. Oceano. and Met., 6 (2), 1-55.

MORGAN, G. W., 1956: On wind-driven ocean circulation. Tellus, 8, 301-320.

Stommel, H., 1958: The abyssal circulation. Deep-Sea. Res. 5, 80-82.
Stommel, H., A. B. Arons and A. J. Faller, 1958: Some examples of stationary planetary flow patterns in bounded basins. Tellus, 10 (2), 175-187.

VeroNIS, G., 1958: On the transient response of a $\beta$-plane ocean. Journ. Oceanog. Soc. Japan, 14 (1), 1-5.

WüsT, G., 1936: Schichtung und Zirkulation des Atlantischen Ozeans. Das Bodenwasser und die Stratosphäre. Wiss. Erg. Deutsch. Atlant. Exp. "Meteor" 1925-26. 6 (1). 